\title{
16. Using Decimal Fractions
}

If a numeric data-item can contain decimal fractions - it must be suitably defined in the Data Division - otherwise only whole numbers will be allowed.

If the item will be used in arithmetic, the position of the decimal point will usually be shown by a ' $V$ '.

$\begin{array}{lllll}\text { e.g. } & 01 \text { WAGE } & \text { PIC 999V99. (or PIC 9(3)V99) }\end{array}$

Note that the decimal point is not stored and therefore does not take up any space in memory - the computer simply takes note of where the point should go so that it can be used in calculations. For this reason, on some computer systems - when entering a number from the keyboard into such a field - the decimal point is not typed: e.g. 323.95 should be keyed as 32395 . Similarly, the decimal point will not be displayed in any number output to the screen - so the user has to remember where it should go.

If you want the decimal point to be displayed - it may be declared as '.'.

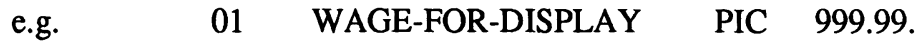

In this case, the decimal point takes up a character of storage - so the above dataitem is 6 characters long.

Arithmetic may not be performed on a field defined in this way (as it is not a numeric field but a 'numeric-edited' field - i.e. a field which has been edited for display purposes) but it can be used for the answer to a calculation (e.g. after GIVING) - or a number may be MOVEd from a numeric field to a numeric-edited field ready for display.

\section{Exercises}

1. Write data definitions for the following fields (which are to be used in arithmetic):
a)
ANNU,
( 7 digits including pence);
b) FLOOR-AREA (maximum size 5000 square feet and the figure will contain one decimal place).

Now write suitable definitions if these fields are to be displayed on the screen with the decimal point shown.

2. Write a program which will accept 2 numbers - each 5 digits including 1 decimal place - add them together and display the answer:

a) with the decimal point assumed (i.e. not actually displayed); and

b) with the decimal point displayed.

3. Write a program which will accept an employee's annual salary (maximum is $£ 20000$ per annum) then calculate and display the weekly wage in the form - for example - 300.25 . 